\title{
An Investigation of the Performance of a Hybrid of Hartree-Fock and Density Functional Theory
}

\author{
PETER M. W. GILL, BENNY G. JOHNSON, and JOHN A. POPLE \\ Department of Chemistry, Carnegie Mellon University, Pittsburgh, Pennsylvania 15213
}

MICHAEL J. FRISCH

Lorentzian Inc., I27 Washington Avenue, North Haven. Connecticut 06473

\begin{abstract}
We explore a hybrid methodology in which the Hartree-Fock energy and density are combined with the nonlocal exchange functional of Becke (1988) and the nonlocal correlation functional of Lee, Yang and Parr (1988) to yield an estimate of the Schrödinger total energy. The resulting mean absolute deviation from a large number of experimental atomization energies, ionization potentials, electron affinities, and proton affinities is $3.86 \mathrm{kcal} / \mathrm{mol}$. Performance comparisons are made with the recently developed G2 theory and nonconventional interpretations of the density functionals are discussed. (c) 1992 John Wiley \& Sons. Inc.
\end{abstract}

\section{Introduction}

Systematic prediction of molecular energy by quantum mechanical methods is one of the principal objectives of theoretical chemistry. In the last few years, there has been some success in developing methods which will reproduce experimental energies, such as dissociation energies, ionization potentials, and electron affinities, to an accuracy of $\pm 2 \mathrm{kcal} / \mathrm{mol}$ or $0.1 \mathrm{eV}$. The GAUSSIAN- 1 (G1) and GAUSSIAN2 (G2) models $[1,2]$ achieve this by ab initio procedures, supplemented by a single small semiempirical parameter to allow for higher-order basis functions and higherorder correlation corrections. However, this level of accuracy is only reached at a cost that rapidly increases with the size of the system (an iterative $N^{6}$ step followed by a single $N^{7}$ step, where $N$ scales as the molecular size). There is, therefore, interest in methods which might reach similar precision more efficiently.

Density functional theory has recently shown promise in this direction. In particular, Becke [3] has proposed an algorithm which, when applied to the same experimental data set as used in Gl theory, gives total atomization energies of neutral molecules with an average absolute error of $3.7 \mathrm{kcal} / \mathrm{mol}$. This result compares with $1.6 \mathrm{kcal} / \mathrm{mol}$ for $\mathrm{G} 1$ and $1.2 \mathrm{kcal} / \mathrm{mol}$ for G2 theory, but is obtained at lower cost and is more easily extendable to larger molecules. Other density functional theories have also shown promise in this direction $[4,5]$.

Most density functional theories attempt to express the exchange and/or correlation energies as functionals of the one-electron density $\rho(r)$ (or of the $\alpha$ - and $\beta$-densities $\rho_{\alpha}(r), \rho_{\beta}(r)$ for open-shell systems). Exact functionals are unknown, 
but some general properties have been elucidated and many explicit forms have been tried. The densities $\rho_{\alpha}, \rho_{\beta}$ are obtained usually via a self-consistent procedure, based on the potential derived from the exchange-correlation functional (KohnSham equations ). However, it is quite possible to apply the same energy functionals to electron densities derived by standard (HF) theory. An extensive study of this sort, using several functionals, has been published by Clementi and Chakravorty [6]. Such comparative studies should help our understanding of both the densities and functionals as well as pointing the way towards improved treatments.

Our principal objective is to apply a combination of energy functionals from the recent literature to a large set of Hartree-Fock densities. This set consists of the atoms, molecules, and ions used in the $G 2$ study [2], where they were tested against well-known atomization energies, ionization potentials, electron affinities, and proton affinities. Here we make a parallel study, using a Hartree-Fock density and a combination of exchange-correlation functionals due to Becke [7] and Lee et al. [8]. Emphasis is on the development of a unique procedure for all species, with well-defined total energies, which may be compared directly with Schrödinger total energies, insofar as they are known. Such total energies, which are rarely reported in the current density functional literature, can then be used to calculate energy differences, directly comparable to experimental data (as is possible with G2 theory).

\section{Method}

In order to facilitate comparison with previous calculations, we shall use molecular structures and frequencies exactly as in G2 theory. All geometrical parameters are found at the MP2/6-31G(d) level and harmonic frequencies at HF/6-31G(d). These frequencies (scaled by the usual 0.893 empirical factor [9]) are used to calculate zero-point vibrational energies. Single-point Hartree-Fock calculations are then carried out with the largest basis used in G2 theory, $6-311+\mathrm{G}(3 d f, 2 p)$ [10]. Restricted (RHF) and unrestricted Hartree-Fock (UHF) methods are used for closed and open shell systems, respectively. The electron density, $\rho$, may be described in conventional notation as $\mathrm{HF} / 6-311+\mathrm{G}(3 d f, 2 p) / / \mathrm{MP} 2 / 6-31 \mathrm{G}(d)$. This is then used for density functional calculations.

In density functional theory, the total energy is written

$$
E=E_{T}+E_{V}+E_{J}+E_{X C}
$$

where $E_{T}$ is the kinetic energy of independent electrons having the density $\rho, E_{V}$ is the potential energy involving nuclei (nuclear-electron + nuclear-nuclear) and $E_{J}$ is the overall coulomb repulsive energy

$$
E_{J}=\frac{1}{2} \iint \rho\left(r_{1}\right) r_{12}^{-1} \rho\left(r_{2}\right) d r_{1} d r_{2}
$$

The remaining term $E_{X C}$ is the exchange-correlation energy, representing the energy lowering due to the fact that the complete electron-electron interaction is less than $E_{J}$, partly because this incorrectly includes the interaction of a particular electron with its own smoothed distribution, and partly because the relative motion of other electrons is correlated, not independent as implicit in Eq. (2). 
In Hartree-Fock theory, the energy is calculated as the expectation value of the Hamiltonian using the optimized single-determinant wavefunction with occupied spin orbitals $\chi_{i}$. This gives an energy

$$
E_{\mathrm{HF}}=E_{T}+E_{V}+E_{J}+E_{X}
$$

where $E_{X}$ is the exchange energy

$$
E_{X}=-\frac{1}{2} \sum_{i, j}^{o c c} \iint \chi_{i}(1) \chi_{j}(1) r_{12}^{-1} \chi_{i}(2) \chi_{j}(2) d \tau_{1}^{i} d \tau_{2}
$$

integration being over cartesian and spin coordinates. This expression takes some account of the tendency of electrons of parallel spin to be kept spatially apart by virtue of the antisymmetry principle. However, as is well known, the Hartree-Fock energy $E_{\mathrm{HF}}$ takes no account of the correlation between electrons of antiparallel spin. The remaining part of the energy is the correlation energy $E_{C}$, which deals with $\alpha-\beta$ correlation and also the remaining $\alpha-\alpha$ and $\beta-\beta$ correlation effects not included in the exchange part of $E_{\mathrm{HF}} . E_{C}$ is difficult and frequently expensive to compute. In G2 theory, it is treated by a combination of Møller-Plesset (MP) and quadratic configuration interaction methods.

A common recommendation in density functional theory is to treat $E_{X}$ and $E_{C}$ together as a single correction $E_{X C}$, determined by a functional $E_{X C}[\rho]$. There is some point to this, since the exchange energy by itself is not clearly defined for densities other than Hartree-Fock. Nevertheless, in practice, $E_{X C}$ often is split into two parts, one of which has the appearance of an exchange correction and the other the appearance of a correlation correction. However, the two do not necessarily correspond to exact exchange and correlation, in the normal meaning of these terms. Ziegler [11], for example, notes that poor results are often obtained if exact (i.e., HF) exchange energies are used in conjunction with certain functionals for correlation. Instead, it is better to use a different density functional theory (DFT) functional for exchange to go along with the correlation calculation. Since the exchange energy is well-defined (at least for $\rho_{\mathrm{HF}}$ ), we prefer to denote this partition of $E_{X C}$ as a division into a parallel-spin $(\alpha \alpha+\beta \beta)$ part $E_{P}$ and an antiparallel$\operatorname{spin}(\alpha \beta)$ part $E_{A}$,

$$
E_{X C}=E_{P}\left[\rho_{\alpha}\right]+E_{P}\left[\rho_{\beta}\right]+E_{A}\left[\rho_{\alpha}, \rho_{\beta}\right]
$$

The parallel part, which is exchange-like, is the sum of an $\alpha$-and a $\beta$-part, determined solely by their respective densities $\rho_{\alpha}$ and $\rho_{\beta}$. The antiparallel part, which is correlation-like, is a single functional $E_{A}\left[\rho_{\alpha}, \rho_{\beta}\right]$ which vanishes unless $\rho_{\alpha}$ and $\rho_{\beta}$ are both nonvanishing and overlapping. A possible interpretation is that $E_{P}$ takes account of both elimination of self-interaction and effects of full correlation of parallel $(\alpha \alpha+\beta \beta)$ electrons (including exchange), while $E_{A}$ takes into account of the remaining correlation between antiparallel $(\alpha \beta)$ electrons. We shall return to this hypothesis in a later section.

We now turn to specification of the functionals. For the parallel part, we use the expression introduced by Becke in 1988 [7], and also used by Becke [3] in his recent study of atomization energies. We shall write this as 


$$
E_{P}^{\mathrm{B8} 8}=E_{P}\left[\rho_{\alpha}\right]+E_{P}\left[\rho_{\beta}\right]
$$

where

$$
\begin{gathered}
E_{P}[\rho]=-\frac{3}{2}\left(\frac{3}{4 \pi}\right)^{1 / 3} \int \rho^{4 / 3} d r-\int \rho^{4 / 3} \frac{b x^{2}}{1+6 b x \sinh ^{-1} x} d r \\
x=\rho^{-4 / 3}|\nabla \rho|
\end{gathered}
$$

The first term in (7) is designed to reproduce the exchange energy of a uniform electron gas. The second term introduces a correction for nonuniformity through the density gradient $\nabla \rho$, the particular analytic form being such that correct asymptotic behaviour is achieved far from the molecular centre. The parameter $b$ is chosen by Becke as $\mathbf{0 . 0 0 4 2}$ to fit the known exchange energies for the inert gas atoms.

The Becke functional (7) is parameterized to give good atomic exchange energies. However, as noted above, it may not always lead to good exchange energies for molecules. It is useful to introduce a quantity

$$
\Delta E_{P}^{\mathrm{B} 88}=E_{P}^{\mathrm{B} 88}-E_{X}
$$

which measures the "Becke 88 excess," or excess exchange energy implied by (7). As will be seen in the next section, this excess is quite large for many molecules. Since we are using a Hartree-Fock density, the values of $E_{P}^{\mathrm{B} 88}$ and $E_{X}$ are both computable and $\Delta E_{P}^{\mathrm{B} 88}$ is easily obtained.

It remains to add the antiparallel correlation correction $E_{A}\left[\rho_{\alpha}, \rho_{\beta}\right]$. Here we use the form introduced by Lee, Yang and Parr (LYP) [8]. This originates in an older study of $\alpha \beta$ correlation in the helium atom by Colle and Salvetti [12]. The actual form programmed is one equivalent to LYP presented by Miehlich, Savin, Stoll, and Preuss (MSSP) [13]. It is given fully by eq. (2) of this reference and need not be reproduced in full. It does have the property of vanishing if either $\rho_{\alpha}$ or $\rho_{\beta}$ is nonexistent, so has the appearance of representing only $\alpha \beta$ correlation. We denote it by $E_{A}^{\text {LYP }}$.

It should be noted that both LYP and MSSP applied the LYP functional to a number of other atomic and molecular systems and sometimes obtained good results when compared with known total correlation energies. Since $E_{A}^{\text {LYP }}$ does not incorporate effects of $\alpha \alpha$ or $\beta \beta$ correlation in any direct manner, the significance of this is somewhat unclear. We shall use $E_{A}^{\mathbf{L Y P}}$ only to represent the antiparallel part.

The (Becke $88+$ Lee, Yang and Parr) correlation energy is then given by

$$
E_{C}^{\mathrm{BLYP}}=\Delta E_{P}^{\mathrm{BB8}}+E_{A}^{\mathrm{LYP}}
$$

and the corresponding total energy $E_{0}^{\mathrm{BLYP}}$ is obtained by adding the HF and zeropoint vibrational energies

$$
E_{0}^{\mathrm{BLYP}}=E_{\mathrm{HF}}+E_{C}^{\mathrm{BLYP}}+E_{\mathrm{ZPV}}
$$


The actual procedure is:

1. Obtain the Hartree-Fock energy $E_{\mathrm{HF}}$;

2. Remove the Hartree-Fock exchange energy $E_{X}$;

3. Compute $E_{P}^{\mathrm{BgB}}$ and $E_{A}^{\mathrm{LYP}}$ by numerical quadrature and add to give $E_{e}^{\mathrm{BLYP}}$;

4. Compute $E_{Z P V}$ and add to give $E_{0}^{\mathrm{sLPP}}$.

Details of the numerical integration scheme are given in the Appendix.

\section{Results and Discussion}

The BLYP procedure just described has been applied to the 152 atoms, molecules, and ions needed for comparison with the experimental G2 data set (which we have extended here to include $\mathrm{H}_{2}, \mathrm{H}_{3}^{+}, \mathrm{He}, \mathrm{He}^{+}, \mathrm{Ne}, \mathrm{Ne}^{+}$, Ar, and $\mathrm{Ar}^{+}$) using a modified version of the GAUSSIAN 92 suite of programs [14]. The source data listed in Table I are:

1. The proper (unrestricted Hartree-Fock) exchange energy, $E_{X}$, with the large 6$311+\mathrm{G}(3 d f, 2 p)$ basis;

2. The Becke-88 excess, $\Delta E_{P}^{\mathrm{B} 88}$, given by Eq. (9), measuring the amount by which the Becke functional (7) gives an energy contribution additional to $E_{X}$;

3. The estimate of the total correlation energy, $E_{C}^{\text {BLYp }}$, given in this theory as the sum of $\Delta E_{P}^{\mathrm{B} 88}$ and the antiparallel part due to Lee, Yang and Parr;

4. The total energy, $E_{0}^{\mathrm{BLYP}}$, obtained by adding the correlation to the Hartree-Fock energy, to give $E_{e}$ and then further modified by addition of the zero-point energy, as listed elsewhere [1,2].

This set of data should prove of value in assessing the role of various energy contributions; it is more comprehensive than most of the data published in the density functional literature.

We begin with information about the total energies of atoms. For small atoms, the total energy (corresponding to full solution of the Schrödinger equation) is moderately well known, either by high-level theory, or by some combination of experimental and theoretical ionization potentials. The BLYP results are compared with some such values in Table II. Agreement is generally achieved within 10 millihartrees $(\mathrm{mh})$, although there is some variation. The Becke-88 functional fails to give the correct exchange energy for the hydrogen atom $(-312.5 \mathrm{mh})$ by $2.8 \mathrm{mh}$, leading to a significant error of $3 \mathrm{mh}$. For the heavier atoms, such as oxygen, fluorine, and neon, there are some partly compensating errors. On the one hand, incompleteness of the orbital basis results in a Hartree-Fock energy that is too positive by about $20 \mathrm{mh}$ for the neon atom (based on the HF limit of -128.5470 given by Veillard and Clementi [15]). On the other hand, the magnitude of the correlation energy is overestimated in this treatment. Here, we have $-31.1 \mathrm{mh}$ from $\Delta E_{P}^{\mathrm{B} 88}$ and $-383.4 \mathrm{mh}$ from $E_{A}^{\mathrm{BLYP}}$, giving a total of $-414.5 \mathrm{mh}$. The actual correlation energy of neon is close to $-390 \mathrm{mh}[16]$.

Table III lists the total atomization energies, $\Sigma D_{0}$, from the present theory, together with G2 and experimental data. This consists of the 55 molecules of the G2 set plus $\mathrm{H}_{2}$. The mean absolute difference between BLYP and experiment is 3.94 $\mathrm{kcal} / \mathrm{mol}$, which may be compared with $1.16 \mathrm{kcal} / \mathrm{mol}$ for $\mathrm{G} 2$ theory. The BLYP 
TABLE I. Source data.

\begin{tabular}{|c|c|c|c|c|c|c|c|c|c|}
\hline Molecule & $E_{X}(\mathrm{mh})$ & $\begin{array}{l}\Delta E_{P}^{\mathbf{B g 8}} \\
(\mathrm{mh})\end{array}$ & $\begin{array}{l}E_{C}^{\text {BLYP }} \\
(\mathrm{mh})\end{array}$ & $E_{0}^{\text {BLYP }}(\mathrm{ht})$ & Molecule & $E_{X}(\mathrm{mh})$ & $\begin{array}{l}\Delta E_{P}^{\mathbf{B B 8}} \\
(\mathrm{mh})\end{array}$ & $\begin{array}{l}E_{C}^{\mathbf{B L Y P}} \\
\text { (mh) }\end{array}$ & $E_{0}^{\text {BLYF }}$ (ht) \\
\hline $\mathbf{H}$ & -312.5 & +2.8 & +2.8 & -0.4970 & & & & & \\
\hline $\mathrm{He}$ & -1026.2 & +1.0 & -42.8 & -2.9027 & $\mathrm{He}^{+}$ & -623.2 & +5.2 & +5.2 & -1.9929 \\
\hline $\mathbf{L i}$ & -1781.0 & +5.9 & -47.5 & -7.4796 & $\mathrm{Li}^{+}$ & -1651.1 & +6.2 & -41.4 & -7.2772 \\
\hline $\mathrm{Be}$ & -2666.2 & +9.0 & -85.5 & -14.6575 & $\mathrm{Be}^{+}$ & -2504.9 & +9.5 & -51.6 & -14.3278 \\
\hline B & -3768.6 & +9.2 & -115.7 & -24.6467 & $\mathrm{~B}^{+}$ & -3491.3 & +10.2 & -96.4 & -24.3320 \\
\hline $\mathrm{C}$ & -5074.6 & +8.7 & -149.6 & -37.8398 & $\mathrm{C}^{+}$ & -4745.2 & +9.8 & -129.4 & -37.4224 \\
\hline $\mathbf{N}$ & -6603.5 & +10.0 & -181.9 & -54.5808 & $\mathrm{~N}^{+}$ & -6212.0 & +12.1 & -161.2 & -54.0488 \\
\hline $\mathrm{O}$ & -8212.3 & -6.0 & -262.7 & -75.0721 & $\mathrm{O}^{+}$ & -7911.6 & +18.3 & -188.4 & -74.5550 \\
\hline$F$ & -10037.0 & -20.3 & -341.4 & -99.7432 & $\mathrm{~F}^{+}$ & -9670.6 & +3.2 & -269.8 & -99.0946 \\
\hline $\mathrm{Ne}$ & -12098.3 & -31.1 & -414.5 & -128.9411 & $\mathrm{Ne}^{+}$ & -11656.5 & -7.1 & -344.6 & -128.1457 \\
\hline $\mathrm{Na}$ & -14017.6 & -12.4 & -420.8 & -162.2668 & $\mathrm{Na}^{+}$ & -13902.2 & -8.1 & -407.4 & -162.0717 \\
\hline $\mathrm{Mg}$ & -15991.7 & -1.0 & -460.5 & -200.0671 & $\mathbf{M g}^{+}$ & -15859.7 & +3.5 & -423.8 & -199.7877 \\
\hline $\mathrm{Al}$ & -18090.4 & +8.0 & -485.1 & -242.3591 & $\mathrm{Al}^{+}$ & -17892.2 & +3.1 & -477.7 & -242.1457 \\
\hline $\mathrm{Si}$ & -20303.1 & +12.2 & -517.0 & -289.3690 & $\mathrm{Si}^{+}$ & -20071.1 & +8.3 & -506.6 & -289.0779 \\
\hline $\mathbf{P}$ & -22641.0 & +15.1 & -551.2 & -341.2604 & $\mathrm{P}^{+}$ & -22368.4 & +10.7 & -540.3 & -340.8855 \\
\hline $\mathbf{S}$ & -25033.0 & +18.3 & -609.4 & -398.1123 & $\mathrm{~S}^{+}$ & -24791.3 & +18.2 & -569.3 & -397.7331 \\
\hline $\mathrm{Cl}$ & -27541.1 & +19.9 & -669.9 & -460.1471 & $\mathrm{Cl}^{+}$ & -27262.2 & +20.4 & -629.0 & -459.6734 \\
\hline Ar & -30183.4 & +25.9 & -724.9 & -527.5318 & $\mathrm{Ar}^{+}$ & -29854.7 & +25.2 & -685.7 & -526.9571 \\
\hline \multirow[t]{2}{*}{$\mathrm{C}^{-}$} & -5265.3 & -5.2 & -176.1 & -37.8833 & $\mathrm{Si}^{-}$ & -20455.1 & +9.9 & -528.8 & -289.4119 \\
\hline & & & & & $\mathbf{P}^{-}$ & -22766.0 & +7.9 & -591.9 & -341.2884 \\
\hline $\mathrm{O}^{-}$ & -8387.7 & -42.0 & -339.4 & -75.1274 & $\mathbf{S}^{-}$ & -25194.6 & +9.9 & -652.4 & -398.1876 \\
\hline$F$ & -10276.6 & -60.2 & -421.2 & -99.8669 & $\mathrm{Cl}^{-}$ & -27748.0 & +12.1 & -712.4 & -460.2778 \\
\hline $\mathrm{H}_{2}$ & -659.7 & +3.1 & -35.3 & -1.1588 & $\mathrm{CN}$ & -11757.7 & -36.9 & -450.4 & -92.6806 \\
\hline $\mathbf{L i H}$ & -2140.2 & +7.3 & -81.1 & -8.0642 & $\mathrm{HCN}$ & -12013.2 & -53.7 & -515.9 & -93.4024 \\
\hline BeH & -3084.3 & +16.2 & -91.1 & -15.2387 & $\mathrm{CO}$ & -13301.6 & -62.4 & -545.4 & -113.3162 \\
\hline $\mathrm{CH}$ & -5423.0 & +5.5 & -190.4 & -38.4649 & $\mathrm{HCO}$ & -13685.4 & -59.2 & -564.7 & -113.8419 \\
\hline $\mathrm{CH}_{2}\left({ }^{3} \mathbf{B}_{1}\right)$ & -5874.6 & +14.8 & -196.4 & -39.1166 & $\mathrm{H}_{2} \mathrm{CO}$ & -14071.2 & -49.6 & -588.9 & -114.4723 \\
\hline $\mathrm{CH}_{2}\left({ }^{1} \mathrm{~A}_{1}\right)$ & -5789.6 & +6.3 & -226.1 & -39.1020 & $\mathrm{H}_{3} \mathrm{COH}$ & -14843.6 & -22.9 & -618.3 & -115.6567 \\
\hline $\mathrm{CH}_{3}$ & -6227.3 & +15.7 & -240.6 & -39.7895 & $\mathrm{~N}_{2}$ & -13050.9 & -79.8 & -560.6 & -109.5275 \\
\hline $\mathrm{CH}_{4}$ & -6592.4 & +17.6 & -276.4 & -40.4460 & $\mathrm{H}_{2} \mathrm{NNH}_{2}$ & -14623.2 & -33.2 & -629.3 & -111.8009 \\
\hline NH & -6936.7 & -1.6 & -236.8 & -55.2100 & NO & -14736.1 & -83.2 & -609.2 & -129.9006 \\
\hline $\mathrm{NH}_{2}$ & -7286.4 & -9.4 & --287.2 & -55.8548 & $\mathrm{O}_{2}$ & -16291.3 & -115.7 & -682.9 & -150.3423 \\
\hline $\mathrm{NH}_{3}$ & -7661.6 & -13.3 & -330.9 & $-\$ 6.5160$ & HOOH & -17063.7 & -103.6 & -739.1 & -151.5425 \\
\hline $\mathrm{OH}$ & -8557.3 & -24.5 & -324.2 & -75.7339 & $F_{2}$ & -19936.3 & -138.7 & -813.4 & -199.5522 \\
\hline $\mathrm{OH}_{2}$ & -8930.3 & -38.0 & -378.2 & -76.4142 & $\mathrm{CO}_{2}$ & -21591.1 & -113.1 & -901.6 & -188.5912 \\
\hline FH & -10411.9 & -45.9 & -408.2 & -100.4547 & $\mathrm{Na}_{2}$ & -28022.9 & -27.4 & -868.0 & -324.5593 \\
\hline $\mathrm{SiH}$ & -20652.8 & +17.5 & -545.7 & -289.9765 & $\mathrm{Si}_{2}$ & -40558.6 & -6.1 & -1101.4 & -578.8468 \\
\hline $\mathrm{SiH}_{2}\left({ }^{1} \mathrm{~A}_{1}\right)$ & -21007.6 & +22.9 & -573.8 & -290.5898 & $\mathbf{P}_{2}$ & -45181.0 & -16.4 & -1227.8 & -682.7032 \\
\hline $\mathrm{SiH}_{2}\left({ }^{3} \mathrm{~B}_{1}\right)$ & -21053.3 & +30.8 & -543.5 & -290.5525 & $S_{2}$ & -50051.0 & +1.8 & -1301.0 & $-796.385 i$ \\
\hline $\mathrm{SiH}_{3}$ & -21413.4 & +39.9 & -573.2 & -291.1907 & $\mathrm{Cl}_{2}$ & -55078.7 & +14.3 & -1398.1 & -920.3803 \\
\hline $\mathrm{SiH}_{4}$ & -21780.7 & +49.8 & -598.6 & -291.8275 & $\mathrm{NaCl}$ & -41688.8 & +8.7 & -1123.5 & -622.5593 \\
\hline $\mathbf{P H}$ & -22984.8 & +19.7 & -583.1 & -341.8698 & $\mathrm{SiO}$ & -28548.5 & -55.6 & -906.8 & -364.7374 \\
\hline $\mathrm{PH}_{2}$ & -23328.5 & +23.0 & -617.2 & -342.4866 & $\mathrm{PO}$ & -30807.7 & -60.7 & -952.0 & -416.5567 \\
\hline $\mathrm{PH}_{3}$ & -23682.7 & +27.6 & -647.7 & -343.1085 & $\mathrm{SC}$ & -30084.0 & -23.3 & -869.9 & -436.2151 \\
\hline SH & -25371.7 & +17.7 & -647.5 & -398.7414 & SO & -33218.2 & -54.4 & -990.6 & -473.3827 \\
\hline $\mathrm{SH}_{2}$ & -25720.4 & +18.0 & -683.2 & -399.3773 & $\mathrm{ClO}$ & -35736.7 & -31.5 & -1019.8 & -535.3139 \\
\hline $\mathrm{ClH}$ & -27889.4 & +15.6 & -711.0 & -460.8045 & $\mathrm{ClF}$ & -37554.7 & -52.6 & -1098.2 & -559.9862 \\
\hline $\mathrm{Li}_{2}$ & -3560.7 & +12.2 & -120.6 & -14.9903 & $\mathrm{Si}_{2} \mathrm{H}_{6}$ & -42883.6 & +89.9 & -1170.4 & -582.4896 \\
\hline $\mathrm{LiF}$ & -11994.2 & -43.3 & -461.2 & -107.4382 & $\mathrm{CH}_{3} \mathrm{Cl}$ & -33809.1 & +28.6 & -953.5 & -500.0574 \\
\hline $\mathrm{HCCH}$ & -10963.1 & -22.4 & -465.2 & -77.2852 & $\mathrm{CH}_{3} \mathrm{SH}$ & -31641.5 & +30.4 & -927.4 & -438.6342 \\
\hline $\mathrm{H}_{2} \mathrm{CCH}_{2}$ & -11743.2 & +5.5 & -491.5 & -78.5046 & $\mathrm{HOCl}$ & -36077.7 & -43.3 & -1067.7 & -535.9644 \\
\hline $\mathrm{H}_{3} \mathrm{CCH}_{3}$ & -12514.1 & +32.7 & -518.5 & -79.7048 & $\mathrm{SO}_{2}$ & -41427.7 & -125.5 & -1377.1 & -548.6482 \\
\hline
\end{tabular}


TABLE I. (Continued)

\begin{tabular}{|c|c|c|c|c|c|c|c|c|c|}
\hline Molecule & $E_{X}(\mathrm{mh})$ & $\begin{array}{c}\Delta E_{P}^{\mathrm{Bg8}} \\
(\mathrm{mh})\end{array}$ & $\begin{array}{c}E_{C}^{\text {BLYP }} \\
\text { (mh) }\end{array}$ & $E_{0}^{\mathrm{BLYP}}(\mathrm{ht})$ & Molecule & $E_{X}(\mathrm{mh})$ & $\begin{array}{l}\Delta E_{P}^{\mathrm{Bgs}} \\
(\mathrm{mh})\end{array}$ & $\begin{array}{l}E_{C}^{\text {BLYP }} \\
\text { (mh) }\end{array}$ & $E_{0}^{\mathrm{BLYP}}(\mathrm{ht})$ \\
\hline $\mathrm{H}_{3}^{+}$ & -712.8 & +3.2 & -35.0 & -1.3145 & $\operatorname{SH}_{2}^{+}\left({ }^{2} A_{1}\right)$ & -25443.6 & +14.6 & -649.9 & -398.9182 \\
\hline $\mathrm{CH}_{4}^{+}$ & -6267.4 & +12.1 & -245.3 & -39.9922 & $\mathrm{SH}_{3}^{+}$ & -25825.0 & +22.7 & -678.2 & -399.6427 \\
\hline $\mathrm{NH}_{3}^{+}$ & -7405.9 & +2.2 & -275.9 & -56.1456 & $\mathrm{ClH}^{+}$ & -27605.1 & +17.2 & -671.3 & -460.3416 \\
\hline $\mathrm{NH}_{4}^{+}$ & -7772.7 & -3.3 & -321.5 & -56.8355 & $\mathrm{ClH}_{2}^{+}$ & -27958.1 & +14.9 & -710.9 & -461.0148 \\
\hline $\mathrm{OH}^{+}$ & -8254.7 & -4.4 & -258.0 & -75.2509 & $\mathrm{HCCH}^{+}$ & -10693.0 & -12.1 & -415.8 & -76.8770 \\
\hline $\mathrm{OH}_{2}^{+}$ & -8617.9 & -20.2 & -318.8 & -75.9544 & $\mathrm{HCHCH}^{+}$ & -11058.1 & -11.8 & -454.4 & -77.5290 \\
\hline $\mathrm{OH}_{3}^{+}$ & -9010.7 & -30.8 & -371.3 & -76.6726 & $\mathrm{H}_{2} \mathrm{CCH}_{2}^{+}$ & -11491.0 & +20.6 & -436.9 & -78.1279 \\
\hline $\mathrm{FH}^{+}$ & -10042.3 & -24.6 & -343.5 & -99.8670 & $\mathrm{CO}^{+}$ & -13074.2 & -60.5 & -493.9 & -112.7915 \\
\hline $\mathrm{SiH}_{4}^{+}$ & -21493.4 & +34.9 & -575.4 & -291.4343 & $\mathrm{~N}_{2}^{+}\left({ }^{2} \Sigma_{2}\right)$ & -12627.8 & -124.5 & -565.7 & -108.9565 \\
\hline $\mathrm{SiH}_{5}^{+}$ & -21858.4 & +45.9 & -599.6 & -292.0725 & $\mathrm{~N}_{2}^{+}\left({ }^{2} \Pi_{u}\right)$ & -12672.8 & -79.2 & -516.3 & -108.9239 \\
\hline $\mathrm{PH}^{+}$ & -22712.6 & +14.0 & -572.2 & -341.5041 & $\mathrm{O}_{2}^{+}$ & -15805.1 & -162.8 & -701.6 & -149.8867 \\
\hline $\mathrm{PH}_{2}^{+}$ & -23062.8 & +17.9 & -602.6 & -342.1292 & $P_{2}^{+}$ & -44940.9 & -16.8 & -1193.3 & -682.3300 \\
\hline $\mathrm{PH}_{\mathbf{3}}^{+}$ & -23468.1 & +34.3 & -604.8 & -342.7509 & $\mathrm{~S}_{2}^{+}$ & -49725.4 & -25.8 & -1305.4 & -796.0443 \\
\hline $\mathrm{PH}_{*}^{+}$ & -23834.3 & +43.7 & -631.3 & -343.4005 & $\mathrm{Cl}_{2}^{+}$ & -54756.5 & -9.9 & -1389.6 & -919.9705 \\
\hline $\mathrm{SH}^{+}$ & -25132.4 & +20.2 & -605.6 & -398.3625 & $\mathrm{CIF}^{+}$ & -37256.5 & -57.4 & -1067.1 & -559.5236 \\
\hline $\mathrm{SH}_{2}^{+}\left({ }^{2} \mathrm{~B}_{1}\right)$ & -25473.5 & +20.9 & -643.7 & -398.9995 & $\mathrm{SC}^{+}$ & -29976.7 & -0.4 & $-792,4$ & -435.7734 \\
\hline $\mathrm{CH}^{-}$ & -5605.3 & -6.8 & -217.1 & -38.5079 & $\mathrm{SiH}^{-}$ & -20808.6 & +19.4 & -554.4 & -290.0130 \\
\hline $\mathrm{CH}_{2}^{-}$ & -5954.2 & -7.6 & -257.7 & -39.1421 & $\mathrm{SiH}_{2}^{-}$ & -21161.9 & +24.9 & -585.0 & -290.6231 \\
\hline $\mathrm{CH}_{3}^{-}$ & -6324.1 & -6.1 & -293.6 & -39.7860 & $\mathrm{SiH}_{3}^{-}$ & -21525.1 & +30.9 & -613.0 & -291.2379 \\
\hline $\mathrm{NH}^{-}$ & -7060.3 & -31.7 & -304.5 & -55.2229 & $\mathrm{PH}^{-}$ & -23107.9 & +12.3 & -623.5 & -341.9039 \\
\hline $\mathrm{NH}_{2}^{-}$ & -7420.9 & -38.3 & -350.3 & -55.8777 & $\mathrm{PH}_{2}^{-}$ & -23459.1 & +15.8 & -654.8 & -342.5264 \\
\hline $\mathrm{OH}^{-}$ & -8736.9 & -60.1 & -396.7 & -75.7963 & $\mathrm{SH}^{-}$ & -25537.2 & +9.3 & -688.4 & -398.8217 \\
\hline $\mathrm{O}_{2}^{-}$ & -16524.2 & -125.7 & -725.7 & -150.3576 & $\mathrm{PO}^{-}$ & -31051.7 & -45.6 & -954.7 & -416.5931 \\
\hline $\mathrm{NO}^{-}$ & -14985.7 & -79.1 & -624.0 & -129.9011 & $S_{2}^{-}$ & -50242.9 & +5.5 & -1326.0 & -796.4407 \\
\hline $\mathrm{CN}^{-}$ & -11848.8 & -56.9 & -516.2 & -92.8482 & $\mathrm{Cl}_{2}^{-}$ & -55271.4 & +10.2 & -1417.2 & -920.4807 \\
\hline
\end{tabular}

TABLE II. Energies for neutral atoms ${ }^{\mathbf{a}}$.

\begin{tabular}{lcccc}
\hline \hline Atom & $E$ (UHF) & $E$ (BLYP) & $E(\text { Sch })^{\mathrm{b}}$ & $\Delta E$ (BLYP-Sch) \\
\hline $\mathrm{H}$ & -0.4998 & -0.4970 & -0.5000 & +3.0 \\
$\mathrm{He}$ & -2.8599 & -2.9026 & -2.9042 & +1.6 \\
$\mathrm{Li}$ & -7.4320 & -7.4796 & -7.4781 & -1.5 \\
$\mathrm{Be}$ & -14.5719 & -14.6574 & -14.6673 & +9.9 \\
$\mathrm{~B}$ & -24.5311 & -24.6467 & -24.6539 & +7.2 \\
$\mathrm{C}$ & -37.6903 & -37.8399 & -37.8451 & +5.2 \\
$\mathrm{~N}$ & -54.3989 & -54.5809 & -54.5895 & +8.6 \\
$\mathrm{O}$ & -74.8093 & -75.0721 & -75.0673 & -4.8 \\
$\mathrm{~F}$ & -99.4018 & -99.7432 & -99.7313 & -11.9 \\
$\mathrm{Ne}$ & -128.5266 & -128.9411 & -128.937 & -4. \\
\hline \hline
\end{tabular}

${ }^{a}$ Total energies in hartrees; differences in millihartrees.

${ }^{b}$ Based on Hartree-Fock and correlation estimates [15], but with the Lamb Shift correction reversed in sign as pointed out [16]. 
mean accuracy is quite close to that obtained by Becke [3], $3.7 \mathrm{kcal} / \mathrm{mol}$, based on the original 55 compounds. He uses a combination of $\mathrm{B} 88$ and a correlation correction based on the paramatrization of the free-electron gas by Vosko et al. [17]. We shall refer to this procedure as $\mathrm{B} 92$.

A detailed examination of the entries in Table III shows little correlation with errors reported for $\mathrm{B} 92$, even though the overall performance is comparable. The BLYP binding energies are mostly too small, whereas B92 tends to overbind. A large BLYP error occurs for the CN radical, for which $D_{0}$ is $13.4 \mathrm{kcal} / \mathrm{mol}$ too low; B92 gives a value $6.1 \mathrm{kcal} / \mathrm{mol}$ too high. This radical is known to be highly spin-contaminated at the UHF level. It is therefore of interest to note that the restricted ROHF density leads to a BLYP binding of $174.0 \mathrm{kcal} / \mathrm{mol}$, in much better agreement with experiment. Other large BLYP errors are found for $\mathrm{C}_{2} \mathrm{H}_{6}$ and $\mathrm{Si}_{2} \mathrm{H}_{6}$, the latter giving an atomization energy $17.2 \mathrm{kcal} / \mathrm{mol}$ too small. These errors are spread over seven bonds, however. $\mathrm{B} 92$ gives good agreement for these molecules.

TABLE III. Total atomization energies $\Sigma D_{0}$ (in $\mathrm{kcal} / \mathrm{mol}$ ).

\begin{tabular}{|c|c|c|c|c|c|c|c|}
\hline Molecule & BLYP & G2 & Expt & Molecule & BLYP & $\mathrm{G} 2$ & Expt \\
\hline $\mathrm{H}_{2}$ & 103.4 & 104.4 & 103.3 & $\mathrm{HCN}$ & 304.2 & 302.8 & 301.8 \\
\hline $\mathrm{LiH}$ & 55.0 & 56.6 & 56.0 & $\mathrm{CO}$ & 253.7 & 258.0 & 256.2 \\
\hline $\mathrm{BeH}$ & 52.8 & 45.5 & 46.9 & $\mathrm{HCO}$ & 271.7 & 271.4 & 270.3 \\
\hline $\mathrm{CH}$ & 80.4 & 80.5 & 79.9 & $\mathrm{H}_{2} \mathrm{CO}$ & 355.4 & 359.3 & 357.2 \\
\hline $\mathrm{CH}_{2}\left({ }^{3} \mathrm{~B}_{1}\right)$ & 177.5 & 178.6 & 179.6 & $\mathrm{H}_{3} \mathrm{COH}$ & 474.9 & 482.3 & 480.8 \\
\hline $\mathrm{CH}_{2}\left({ }^{\prime} \mathrm{A}_{1}\right)$ & 168.3 & 172.0 & 170.6 & $\mathrm{~N}_{2}$ & 229.6 & 223.8 & 225.1 \\
\hline $\mathrm{CH}_{3}$ & 287.8 & 289.1 & 289.2 & $\mathrm{H}_{2} \mathrm{NNH}_{2}$ & 408.7 & 404.4 & 405.4 \\
\hline $\mathrm{CH}_{4}$ & 387.9 & 393.2 & 392.5 & NO & 155.4 & 150.6 & 150.1 \\
\hline NH & 83.0 & 77.9 & 79.0 & $\mathrm{O}_{2}$ & 124.3 & 115.6 & 118.0 \\
\hline $\mathrm{NH}_{2}$ & 175.7 & 170.1 & 170.0 & $\mathrm{HOOH}$ & 253.7 & 252.1 & 252.3 \\
\hline $\mathrm{NH}_{3}$ & 278.7 & 276.5 & 276.7 & $F_{2}$ & 41.3 & 36.6 & 36.9 \\
\hline $\mathrm{OH}$ & 103.4 & 101.6 & 101.3 & $\mathrm{CO}_{2}$ & 381.0 & 384.6 & 381.9 \\
\hline $\mathrm{OH}_{2}$ & 218.4 & 219.6 & 219.3 & $\mathrm{Na}_{2}$ & 16.1 & 19.2 & 16.6 \\
\hline $\mathrm{FH}$ & 134.6 & 136.3 & 135.2 & $\mathrm{Si}_{2}$ & 68.3 & 73.6 & 74.0 \\
\hline $\mathrm{SiH}_{2}\left({ }^{1} \mathrm{~A}_{1}\right)$ & 142.3 & 147.1 & 144.4 & $\mathrm{P}_{2}$ & 114.5 & 114.7 & 116.1 \\
\hline $\mathrm{SiH}_{2}\left({ }^{3} \mathrm{~B}_{1}\right)$ & 118.9 & 123.8 & 123.4 & $\mathrm{~S}_{2}$ & 100.7 & 97.4 & 100.7 \\
\hline $\mathrm{SiH}_{3}$ & 207.5 & 213.5 & 214.0 & $\mathrm{Cl}_{2}$ & 54.0 & 55.8 & 57.2 \\
\hline $\mathrm{SiH}_{4}$ & 295.2 & 304.8 & 302.8 & $\mathrm{NaCl}$ & 91.2 & 98.8 & 97.5 \\
\hline $\mathrm{PH}_{2}$ & 145.7 & 144.9 & 144.7 & $\mathrm{SiO}$ & 185.9 & 188.8 & 190.5 \\
\hline $\mathrm{PH}_{3}$ & 224.1 & 226.4 & 227.4 & $\mathrm{SC}$ & 165.0 & 170.5 & 169.5 \\
\hline $\mathrm{SH}_{2}$ & 170.1 & 173.0 & 173.2 & SO & 124.4 & 120.8 & 123.5 \\
\hline $\mathrm{ClH}$ & 100.7 & 102.6 & 102.2 & $\mathrm{ClO}$ & 59.4 & 61.2 & 63.3 \\
\hline $\mathbf{L i}_{2}$ & 19.5 & 25.9 & 24.0 & ClF & 60.2 & 61.0 & 60.3 \\
\hline LiF & 135.2 & 137.5 & 137.6 & $\mathrm{Si}_{2} \mathrm{H}_{6}$ & 482.9 & 503.0 & 500.1 \\
\hline $\mathrm{HCCH}$ & 383.8 & 387.2 & 388.9 & $\mathrm{CH}_{3} \mathrm{Cl}$ & 363.6 & 372.1 & 371.0 \\
\hline $\mathrm{H}_{2} \mathrm{CCH}_{2}$ & 525.2 & 531.7 & 531.9 & $\mathrm{CH}_{3} \mathrm{SH}$ & 435.6 & 445.0 & 445.1 \\
\hline $\mathrm{H}_{3} \mathrm{CCH}_{3}$ & 654.6 & 666.6 & 666.3 & $\mathrm{HOCl}$ & 155.7 & 156.8 & 156.3 \\
\hline $\mathrm{CN}$ & 163.2 & 176.0 & 176.6 & $\mathrm{SO}_{2}$ & 245.8 & 248.9 & 254.0 \\
\hline
\end{tabular}


Table IV gives ionization energies for the same $\mathrm{G} 2$ set plus values for the hydrogen atom and the three inert gases helium, neon and argon. The mean absolute difference between BLYP and experiment is $0.195 \mathrm{eV}(4.49 \mathrm{kcal} / \mathrm{mol})$, compared with 0.054 $\mathrm{eV}(1.24 \mathrm{kcal} / \mathrm{mol})$ by the $\mathrm{G} 2$ procedure. We note that the majority $(30 \mathrm{of} 42)$ of the BLYP ionization energies are smaller than experiment. In particular, the result for the hydrogen atom is significantly too small (by $0.08 \mathrm{eV}$ ). This may be traced to the failure of the Becke- 88 formula to give the correct exchange energy for the hydrogen atom. On the other hand, the worst error in Table IV is for carbon monosulfide, for which the BLYP value is $0.69 \mathrm{eV}$ too large. Our results show comparable accuracy to a smaller set of ionization energies of another DFT study reported by Ziegler [11], using different functionals.

Table $\mathrm{V}$ lists electron affinities for the G2 dataset. The mean error for BLYP is found to be $0.137 \mathrm{eV}(3.16 \mathrm{kcal} / \mathrm{mol})$, compared with $0.056 \mathrm{eV}(1.29 \mathrm{kcal} / \mathrm{mol})$ for G2 theory. The majority ( 20 of 25 ) of the values are numerically too small. However, the result more seriously in error is the cyano radical, for which the BLYP electron affinity is $0.74 \mathrm{eV}$ too high. This large error evidently is due to the poor description of the neutral radical, as already noted in the discussion of atomization energies. Our results may be compared with a DFT study of electron affinities due to Ziegler and Gutsev [18], using different functionals. They studied many of the same molecules and also found an average error of about $0.2 \mathrm{eV}$. However, for the cyano radical, they obtain an electron affinity which is too small, whereas ours is

TABLE IV. Ionization potentials (eV).

\begin{tabular}{|c|c|c|c|c|c|c|c|}
\hline Molecule & BLYP & $\mathrm{G} 2$ & Expt & Molecule & BLYP & $\mathrm{G} 2$ & Expt \\
\hline $\mathbf{H}$ & 13.52 & 13.60 & 13.60 & $\mathrm{OH}_{2}$ & 12.51 & 12.63 & 12.62 \\
\hline $\mathrm{He}$ & 24.76 & 24.54 & 24.59 & $\mathrm{FH}$ & 15.99 & 16.08 & 16.04 \\
\hline $\mathrm{Li}$ & 5.51 & 5.34 & 5.39 & $\mathrm{SiH}_{4}$ & 10.70 & 11.01 & 11.00 \\
\hline $\mathrm{Be}$ & 8.97 & 9.41 & 9.32 & PH & 9.95 & 10.09 & 10.15 \\
\hline B & 8.56 & 8.20 & 8.30 & $\mathrm{PH}_{2}$ & 9.73 & 9.72 & 9.82 \\
\hline C & 11.36 & 11.18 & 11.26 & $\mathrm{PH}_{3}$ & 9.73 & 9.87 & 9.87 \\
\hline $\mathrm{N}$ & 14.48 & 14.48 & 14.54 & SH & 10.31 & 10.31 & 10.37 \\
\hline $\mathrm{O}$ & 14.07 & 13.52 & 13.61 & $\mathrm{SH}_{2}\left({ }^{2} \mathbf{B}_{1}\right)$ & 10.28 & 10.43 & 10.47 \\
\hline $\mathbf{F}$ & 17.65 & 17.39 & 17.42 & $\mathrm{SH}_{2}\left({ }^{2} \mathrm{~A}_{1}\right)$ & 12.49 & 12.75 & 12.78 \\
\hline $\mathrm{Ne}$ & 21.64 & 21.61 & 21.56 & $\mathrm{ClH}$ & 12.60 & 12.71 & 12.75 \\
\hline $\mathrm{Na}$ & 5.31 & 4.95 & 5.139 & $\mathbf{H C C H}$ & 11.11 & 11.42 & 11.40 \\
\hline $\mathrm{Mg}$ & 7.60 & 7.65 & 7.646 & $\mathrm{H}_{2} \mathrm{CCH}_{2}$ & 10.25 & 10.58 & 10.51 \\
\hline $\mathrm{Al}$ & 5.81 & 5.93 & 5.984 & $\mathrm{CO}$ & 14.28 & 14.01 & 14.01 \\
\hline $\mathrm{Si}$ & 7.92 & 8.10 & 8.15 & $\mathrm{~N}_{2}\left({ }^{2} \Sigma_{\mathrm{g}}\right)$ & 15.54 & 15.56 & 15.58 \\
\hline$P$ & 10.20 & 10.44 & 10.49 & $\mathrm{~N}_{2}\left({ }^{2} \Pi_{u}\right)$ & 16.42 & 16.67 & 16.70 \\
\hline$S$ & 10.32 & 10.20 & 10.36 & $\mathrm{O}_{2}$ & 12.40 & 12.17 & 12.07 \\
\hline $\mathrm{Cl}$ & 12.89 & 12.85 & 12.97 & $P_{2}$ & 10.16 & 10.54 & 10.53 \\
\hline $\mathrm{Ar}$ & 15.64 & 15.69 & 15.76 & $\mathrm{~S}_{2}$ & 9.27 & 9.28 & 9.36 \\
\hline $\mathrm{CH}_{4}$ & 12.35 & 12.68 & 12.62 & $\mathrm{Cl}_{2}$ & 11.15 & 11.51 & 11.50 \\
\hline $\mathrm{NH}_{3}$ & 10.08 & 10.19 & 10.18 & $\mathrm{ClF}$ & 12.59 & 12.65 & 12.66 \\
\hline $\mathrm{OH}$ & 13.14 & 12.98 & 13.01 & $\mathrm{SC}$ & 12.02 & 11.42 & 11.33 \\
\hline
\end{tabular}


TABLE V. Electron affinities (eV).

\begin{tabular}{lcccllll}
\hline \hline Molecule & BLYP & G2 & Expt. & Molecule & BLYP & G2 & Expt. \\
\hline $\mathrm{C}$ & 1.18 & 1.19 & 1.26 & $\mathrm{Si}$ & 1.17 & 1.35 & 1.385 \\
$\mathrm{CH}$ & 1.17 & 1.13 & 1.24 & $\mathrm{SiH}$ & 0.99 & 1.18 & 1.277 \\
$\mathrm{CH}_{2}$ & 0.69 & 0.66 & 0.65 & $\mathrm{SiH}_{2}$ & 0.91 & 0.99 & 1.124 \\
$\mathrm{CH}_{3}$ & -0.10 & 0.04 & 0.08 & $\mathrm{SiH}_{3}$ & 1.28 & 1.42 & 1.44 \\
$\mathrm{CN}$ & 4.56 & 3.97 & 3.82 & $\mathrm{P}$ & 0.76 & 0.64 & 0.746 \\
$\mathrm{NH}$ & 0.35 & 0.30 & 0.38 & $\mathrm{PH}$ & 0.93 & 0.96 & 1.00 \\
$\mathrm{NH}_{2}$ & 0.62 & 0.77 & 0.74 & $\mathrm{PH}_{2}$ & 1.08 & 1.25 & 1.26 \\
$\mathrm{NO}_{\mathrm{O}}$ & 0.01 & -0.07 & 0.02 & $\mathrm{PO}$ & 0.99 & 1.04 & 1.09 \\
$\mathrm{OH}$ & 1.50 & 1.40 & 1.46 & $\mathrm{~S}$ & 2.05 & 2.00 & 2.077 \\
$\mathrm{O}_{2}$ & 1.70 & 1.87 & 1.83 & $\mathrm{SH}$ & 2.19 & 2.30 & 2.314 \\
$\mathrm{~F}$ & 0.42 & 0.47 & 0.44 & $\mathrm{~S}$ & 1.51 & 1.66 & 1.663 \\
& 3.37 & 3.48 & 3.40 & $\mathrm{Cl}$ & 3.56 & 3.60 & 3.615 \\
\hline
\end{tabular}

much too large. Like us, they use spin-unrestricted methods. The reasons for this anomaly are hard to pursue further as Ziegler and Gutsev do not report their detailed energy components for the separate species $\mathrm{CN}$ and $\mathrm{CN}^{-}$.

Table VI gives eight proton affinities that are compared with G2 theory and experiment. Here the performance of BLYP is quite good, the mean error of 2.11 $\mathrm{kcal} / \mathrm{mol}$ being not much greater than the value of $1.04 \mathrm{kcal} / \mathrm{mol}$ obtained at the G2 level. This superior performance is not surprising, since no change in the number of electrons is involved and the electron configurations of the protonated species are closely related to those of the neutral molecules.

Combining all of the 130 experimental comparisons in Tables III to VI, we obtain an overall mean absolute error of $3.86 \mathrm{kcal} / \mathrm{mol}$ for the BLYP model. This is not much greater than the value of $3.7 \mathrm{kcal} / \mathrm{mol}$ obtained by Becke [3] for atomization energies only.

\section{Parallel and Antiparallel Contributions}

We return now to the interpretation of the separation of $E_{X C}$ into parallel and antiparallel parts, $E_{P}^{\mathrm{B} 88}$ and $E_{A}^{\mathrm{tYP}}$. As noted earlier, one possibility is that the parallel

TABLE VI. Proton affinities ( $\mathrm{kcal} / \mathrm{mol})$.

\begin{tabular}{lrrrrrrr}
\hline \hline Molecule & BLYP & G2 & Expt & Molecule & BLYP & G2 & Expt \\
\hline $\mathrm{H}_{2}$ & 97.7 & 99.2 & 100.8 & $\mathrm{SiH}_{4}$ & 153.7 & 153.0 & 154 \\
$\mathrm{HCCH}$ & 153.0 & 153.6 & 152.3 & $\mathrm{PH}_{3}$ & 183.2 & 186.2 & 187.1 \\
$\mathrm{NH}_{3}$ & 200.5 & 202.5 & 202.5 & $\mathrm{H}_{2} \mathrm{~S}$ & 166.5 & 167.7 & 168.8 \\
$\mathrm{H}_{2} \mathrm{O}$ & 162.1 & 163.1 & 165.1 & $\mathrm{HCl}$ & 132.0 & 133.0 & 133.6 \\
\hline
\end{tabular}


part represents the full exchange energy plus further energy lowering due to extra (non-Hartree-Fock) correlation energy between $\alpha \alpha$ and $\beta \beta$ pairs. Inspection of Table I, clearly shows that this cannot be correct in all cases because the Becke- 88 excess $\Delta E_{P}^{\mathrm{Bg} 8}$ is sometimes positive, indicating an energy lowering of less than the exchange energy. This cannot be correct. It shows up for the hydrogen atom, where there is no electron correlation, but the Becke- 88 energy fails to cancel the coulomb energy $E_{J}$, as it should. This may, of course, be a fault of the particular analytic form of the Becke- 88 energy. Indeed, the constant $b$ in Eq. (7) could be increased so that $\Delta E_{P}^{\mathrm{B} 88}$ is zero for the hydrogen atom; all values of $\Delta E_{P}^{\mathrm{B} 88}$ for other systems then become negative. However, we will not investigate modified functionals here.

Another possibility is that the partition of correlation into $\Delta E_{P}^{\mathrm{Bg} 8}$ and $E_{A}^{\mathrm{LYP}}$ gives the breakdown of correlation binding into parallel and antiparallel parts, even though this does not work fully for the isolated atoms. We have tested this idea by evaluating some of these binding contributions from Table I (they turn out all to be positive) and compare them with the corresponding parallel $(\alpha \alpha+\beta \beta)$ and antiparallel $(\alpha \beta)$ contributions from an MP2 treatment (frozen core or valence electrons only with the same basis), where the parallel-antiparallel partition is unequivocal. This comparison as displayed in Table VII shows moderate success for the simple hydrides $\mathrm{CH}_{4}, \mathrm{NH}_{3}, \mathrm{OH}_{2}$, and $\mathrm{FH}$. However, for the two heavy-atom molecules $\mathrm{N}_{2}$ and $\mathrm{F}_{2}$, the comparison is much less successful. For $F_{2}$, the satisfactory reproduction of the total correlation binding (as evidenced by the good dissociation energy in Table III) is ascribed mainly to the parallel (Becke-88) part rather than to the antiparallel (LYP) part. At the MP2 level, the opposite is true. Evidently, the success of the BLYP procedure cannot be fully understood along these lines; new insight is needed.

\section{Conclusions}

The following conclusions are drawn from this work:

1. The BLYP procedure described, based on existing energy functionals and using the Hartree-Fock density, is capable of giving total energies in reasonable agreement $(\sim 20 \mathrm{mh})$ with exact Schrödinger energies, insofar as these are known.

TABLE VII. Parallel and antiparallel binding energies (mh).

\begin{tabular}{lcrrrr}
\hline \hline & \multicolumn{2}{c}{ Parallel } & & \multicolumn{2}{c}{ Antiparallel } \\
\cline { 2 - 3 } \cline { 5 - 6 } Molecule & MP2 $(\alpha \alpha+\beta \beta)$ & $\Delta\left(\Delta E^{\mathrm{B88}}\right)$ & & MP2 $(\alpha \beta)$ & $\Delta\left(E^{\mathrm{LYP}}\right)$ \\
\hline $\mathrm{CH}_{4}$ & 23.4 & 2.9 & 103.4 & 135.8 \\
$\mathrm{NH}_{3}$ & 22.8 & 31.4 & 114.1 & 125.9 \\
$\mathrm{OH}_{2}$ & 28.6 & 37.5 & 89.9 & 83.5 \\
$\mathrm{FH}$ & 21.5 & 28.3 & & 52.0 & 41.3 \\
$\mathrm{~N}_{2}$ & 40.1 & 99.6 & 153.1 & 97.0 \\
$\mathrm{~F}_{2}$ & 34.7 & 97.9 & 90.5 & 32.8 \\
\hline \hline
\end{tabular}


2. Various differences of BLYP total energies reproduce good experimental data on atomization energies, ionization energies, electron affinities and proton affinities to an accuracy of about $4 \mathrm{kcal} / \mathrm{mol}$ or $0.2 \mathrm{eV}$. Even though this does not match the greater accuracy achieved by G2 theory, it is a significant achievement for a method of moderate computational cost. The use of a single, well-defined, theoretical procedure for all of these physical properties gives some coherence to the theory.

3. The partition of the electron correlation into parallel (B88) and antiparallel (LYP) parts does not always match the corresponding partition in simple MøllerPlesset theory. The significance of the individual components of BLYP (or other comparable density functional treatments) is not fully understood.

\section{Acknowledgments}

We are indebted to Dr. A.D. Becke for an advance copy of Ref. 3 and some valuable discussions. We thank Elizabeth Gill for her help in preparing the tables and for typing this manuscript. This research was partly supported by National Science Foundation Grant No. 8918623.

\section{Appendix: Numerical Integration in Three Dimensions}

Our preliminary method for evaluating density functionals such as (7) is closely related to that described by Becke [19] but, for various reasons, we have made a number of modifications to his scheme which we now describe.

(1) Our cell functions $P_{i}(r)$ are identical to Becke's for homonuclear systems, but we have not found it necessary to adopt the "atomic size adjustments" which Becke suggests for heteronuclear systems.

(2) We employ Gauss-Laguerre, rather than Gauss-Chebyshev, quadrature for the single-center radial subintegrations. This choice was motivated by the fact that the true electron density is known to decay exponentially at large distances from the molecule.

(3) We use 31 radial points on each center, scaled so that the 16 th (middle) point lies at the maximum of the radial probability function $4 \pi r^{2} \varphi^{2}(r)$ of the valence atomic orbital $\varphi(\mathrm{r})$ given by Slater's well known rules [20]. For example, the 16th radial point for a hydrogen atom lies at $r=1.0$ au and that for a carbon atom lies at $r=16 / 13 \mathrm{au}$.

(4) We use 72 angular points at each radial point. The distribution of these is given by a special set of 12 points corresponding to the vertices of a regular icosahedron inscribed in the sphere (the axes of the icosahedron follow the "standard orientation" conventions of the GAUSSIAN program), plus a general set of 60 points generated by the rotation subgroup of the icosahedral group. Such formulae, based on finite rotation subgroups of the sphere, are highly efficient in the sense that the number of surface harmonics exactly integrated by them is close to one per angular point. A clear exposition of the theory of 
this approach to quadrature on the surface of a sphere can be found in the literature [21].

If the electron density $\rho(r)$ itself is integrated using this preliminary scheme, the results agree with the total number of electrons to within a few thousandths of an electron for all of the molecules studied. Furthermore, we have observed that both the B88 and LYP functional values are comparatively insensitive to further improvements in the grid. Nonetheless, further work is underway to construct even more efficient integration schemes.

\section{Bibliography}

[1] (a) J. A. Pople, M. Head-Gordon, D. J. Fox, K. Raghavachari, and L. A. Curtiss, J. Chem. Phys. 90, 5622 (1989); (b) L. A. Curtiss, C. Jones, G. W. Trucks, K. Raghavachari, and J. A. Pople, J. Chem. Phys. 93, 2537 (1990).

[2] L. A. Curtiss, K. Raghavachari, G. W. Trucks, and J. A. Pople, J. Chem. Phys. 94, 7221 (1991).

[3] A. D. Becke, J. Chem. Phys. 96, 2155 (1992).

[4] J. Andzelm and E. Wimmer, J. Chem. Phys. 96, 1280 (1992).

[5] B. Delley, J. Chem. Phys. 92, 508 (1990).

[6] E. Clementi and S. J. Chakravorty, J. Chem. Phys. 93, 2591 (1990).

[7] A. D. Becke, Phys. Rev. A38, 3098 ( 1988).

[8] C. Lee, W. Yang, and R. G. Parr, Phys. Rev. B37, 785 (1988).

[9] J. A. Pople, H. B. Schlegel, R. Krishnan, D. J. Defrees, J. S. Binkley, M. J. Frisch, R. A. Whiteside, R. F. Hout, and W. J. Hehre, Int. J. Quantum Chem. Symp. 15, 269 (1981).

[10] Hitherto, the 6-311+G(3df,2p) basis set has not been defined for Ne and Ar. We have therefore defined the diffuse function exponents for $\mathrm{Ne}$ and $\mathrm{Ar}$ to be 0.13 and 0.06 , respectively, and the $\mathrm{f}$ function exponent for Ar to be 0.85 .

[11] T. Ziegler, Chem. Rev. 91, 651 (1991).

[12] R. Colle and O. Salvetti, Theoret. Chim. Acta 37, 329 (1975).

[13] B. Miehlich, A. Savin, H. Stoll, and H. Preuss, Chem. Phys. Letters 157, 200 (1989).

[14] GAUSSIAN 92, M. J. Frisch, G. W. Trucks, M. Head-Gordon, P. M. W. Gill, M. W. Wong, J. B. Foresman, B. G. Johnson, H. B. Schlegel, M. A. Robb, E. S. Replogle, R. Gomperts, J. L. Andres, K. Raghavachari, J. S. Binkley, C. Gonzalez, R. L. Martin, D. J. Fox, D. J. Defrees, J. Baker, J. J. P. Stewart, and J. A. Pople, Gaussian, lnc., Pittsburgh, PA, 1992.

[15] A. Veillard and E. Clementi, J. Chem. Phys. 49, 2415 (1968).

[16] R. K. Nesbet, T. L. Barr, and E. R. Davidson, Chem. Phys. Letters 4, 203 (1969).

[17] S. H. Vosko, L. Wilk, and M. Nusair, Can. J. Phys. 58, 1200 (1980).

[18] T. Ziegler and G. L. Gutsev, J. Comput. Chem. 13, 70 (1992).

[19] A. D. Becke, J. Chem. Phys. 88, 2547 (1988).

[20] J. C. Slater, Phys. Rev. 36, 57 (1930).

[21] A. D. McLaren, Math. Comput. 17, 361 (1963).

Received April 3, 1992 